\title{
FORMACIÓN DE MAESTROS RURALES COLOMBIANOS 1946-1994
}

\author{
Alba Nidia Triana Ramírez ${ }^{1}$ \\ Universidad Pedagógica y Tecnológica de Colombia \\ educarural@gmail.com
}

Recepción: 18/04/2011

Evaluación: 10/11/2011

Aceptación: 29/05/2012

Artículo de Reflexión

doi: 10,9757

\section{RESUMEN}

El artículo trata sobre la evolución de la política y la práctica relacionada con la formación, capacitación y mejoramiento de los maestros rurales en Colombia durante el período comprendido entre 1946 a 1994. Toma para el análisis cuatro tópicos fundamentales: los maestros rurales durante la segunda hegemonía conservadora, las reformas y estructuras de las Escuelas Normales en la segunda mitad del siglo XX, la capacitación y perfeccionamiento docente, los cursos-créditos y los microcentros rurales. El estudio es hermenéutico, tiene como soporte metodológico fuentes documentales en lo que respecta a planes, programas, leyes y decretos, artículos de revistas y las Memorias del Ministro de Educación al Congreso de la República de Colombia. De la misma manera, se toma para el análisis, fuentes secundarias, fundamentadas en investigaciones realizadas sobre la educación en el período descrito. Se concluye, que la formación, capacitación y mejoramiento de los maestro rurales colombianos, estuvo supeditada a las transformaciones y procesos de modernización estructurados a los largo de los años 1946 - 1994. La formación de maestros y maestras para la educación rural, no se definió desde los contextos socioculturales, que le permitieran enfrentar la dinámica y compleja vida tradicional campesina, por el contrario, se supeditó a las tendencias de un orden mundial cuya estructura ideológica se fundamentaba en la producción, la globalización y una modernización sin modernidad.

Palabras clave: Revista Historia de la educación Latinoamericana, maestros rurales, decretos, políticas educativas, reformas.

\footnotetext{
1 Doctora en Ciencias de la Educación RUDECOLOMBIA, profesora de la Facultad de Ciencias de la Salud de la Universidad Pedagógica y Tecnológica de Colombia, coordinadora del Grupo de investigación de Educación Rural- UPTC, Asesora Académica del Doctorado en Ciencias de la Educación CADE- Tunja.
} 


\section{TEACHING MEHTOD IN COLOMBIAN COUNTRY SIDE EDUCATORS 1946-1994}

\begin{abstract}
This paperwork is about political and practical evolution related to teaching, skill developing and improvement of country side teachers in Colombia in the time between 1946 and 1994. It brings four specific and fundamental topics: Country side educators during the second conservative regime, Reformation and structure of Teaching schools during the second half of 20 th century, skill development and teaching improvement, lessons by credits and micro centers in country side areas.
\end{abstract}

This is an Hermeneutical study, it has a methodollogical support in documents and sources for plans, programms, laws, and articles or bills of colombian constitution, as well as memoirs of Education Minister in the congress chamber of The Republic of Colombia.

In the same line secondary sources were analysed, these were research journals and documents about education during those ages. Article concludes that teaching, proving and testing processes of country side educators was stoped mainly because of change and modernization procedures that were stablished during 1946 til 1994 .

Teaching and profesional tutoring of country side educators (both male and female) was not outlined from soci cultural context that may have allowed face dynamics and complexity of traditional life style of peasants, on the countrary it was well related to global trends which ideological structure was related to production, globalization and modernization without any modernity at all.

Key words: Journal of Latin American Education History country side Educators, articles, education politics, reformation.

\section{FORMAÇÃO DE PROFESSORES COLOMBIANOS 1946-1994}

\section{RESUMO}

O artigo trata sobre a evolução da política e da prática relacionada com a formação, capacitação e melhoramento dos professores rurais na Colômbia durante o período compreendido entre 1946 e 1994. Toma para análise quatro tópicos fundamentais: os professores rurais durante a segunda hegemonia conservadora, as reformas e estruturas das Escolas Normais na segunda metade do século XX, a capacitação e aperfeiçoamento docente, os cursos-créditos e os pequenos centros rurais. O estudo é hermenêutico, tem como suporte metológico fontes documentais no que diz respeito a planos, programas, leis e decretos, artigos de revistas e as Memórias do Ministro da Educação ao Congresso da República da Colômbia. Da mesma maneira, toma-se para análise fontes secundárias, fundamentadas em pesquisas realizadas sobre a educação no período descrito. Conclui-se que a formação, capacitação e melhoramento dos professores rurais colombianos estiveram subordinados às transformações e processos de modernização estruturados ao longo dos anos 1946-1994. A formação de professores e professoras para a educação rural não se definiu desde os contextos que lhe permitiram enfrentar a dinâmica e complexa vida tradicional campesina, pelo contrário, se subordinou às tendências de uma ordem mundial cuja estrutura ideológica se fundamentava na produção, na globalização e em uma modernização sem modernidade.

Palavras-chave: Revista Historia da Educação Latino-americana, professores rurais, decretos, politicas educativas, reformas. 


\section{INTRODUCCIÓN}

[...], por frágiles que hayan sido los procesos formativos, los maestros rurales colombianos, de segunda mitad de siglo XX, desde su praxis pedagógica cotidiana, urdieron finos ligamentos para unir el vasto mundo tradicional de lo rural, con las exigencias del mundo moderno.

Alba Nidia Triana

A propósito del bicentenario de la independencia de Colombia, es pertinente preguntarse por la trayectoria de la educación rural. Hemos de recordar, que este era un país rural, y así lo fue hasta entrada la segunda mitad del siglo XX. Sin embargo, los historiadores se han ocupado por investigar la educación en términos generales, en cierta forma centrados en lo que ha sido la educación desde los ámbitos urbanos, pero se han olvidado de un amplio segmento de la población- la campesina- a la cual la educación llegó y aun sigue llegando de manera tardía, por lo que se constituye en el más explícito mecanismo de exclusión social.

Sibien, con este artículo no se pretende demostrar en su conjunto la trayectoria de la educación rural en estos doscientos años, tarea que sin lugar a dudas es ardua en tiempo y discurso, pues el tema de la exclusión, requiere indagar sobre los hilos invisibles que soportaron las relaciones de poder y enmarcaron, mucho antes de la independencia los procesos sociales, políticos y económicos, sobre los que se fundó la república. Solamente, se hará referencia a la formación de los maestros rurales en el período comprendido entre 1946-1994.

Tiene como objetivo general indagar de manera descriptiva, a cera de la evolución de la política y la práctica relacionada con la formación, capacitación y mejoramiento de los maestros rurales en Colombia durante el período comprendido entre 1946 a 1994, a partir de cuatro tópicos fundamentales: los maestros rurales durante la segunda hegemonía conservadora, las reformas y estructuras de las Escuelas Normales en la segunda mitad del siglo XX, la capacitación y perfeccionamiento docente, los cursos-créditos y los microcentros rurales.

La formación de los maestros en Colombia ha sido tema de interés y preocupación en los diferentes ámbitos, procesos y gobiernos, así como de los organismos internacionales; este tópico se encuentra implícito en la trayectoria de las políticas educativas del país.

Diferentes organismos y conferencias internacionales, como la Organización de los Estados Americanos (OEA), la Organización de las Naciones Unidas para la Educación, la Ciencias y la Cultura (Unesco), las 
Conferencias Interamericanas de Educación y Conferencias Interamericanas de Instrucción Pública, en reiteradas oportunidades, desde 1937 hasta 1960, trataron el tema de la formación y perfeccionamiento del magisterio de primaria, y particularmente se mencionó la formación de maestros rurales.

La Tercera Conferencia Interamericana de Educación (México, agosto de 1937), el Seminario Interamericano de Educación Primaria (Montevideo, septiembre de 1950), la Decimosexta Conferencia Internacional de Instrucción Pública (Ginebra, julio de 1953), la Conferencia Regional sobre Educación Gratuita y Obligatoria en América Latina (Lima, abril de 1956) donde se establece que "la preparación del maestro urbano y rural sea equivalente, sin desvirtuar la preparación específica de cada uno de ellos"2.

En la década de los años sesenta, como resultado del nuevo orden mundial que se enmarco en la ruta del desarrollo económico, obligó a gobiernos y organismos internacionales a plantear reformas educativas y políticas de formación de maestros con un fin específico: contribuir a la expansión del sistema educativo como alternativa principal para la modernización y el desarrollo de la sociedad al estilo occidental, a partir de nuevas estrategias y modelos educativos.

Los albores de la segunda mitad del siglo XX vinieron acompañados de los primeros diagnósticos socioeconómicos realizados en América Latina, de los cuales Colombia se convirtió en pionero. El balance permitió reflexionar acerca de la gran deficiencia que el país tenía en materia de educación. En consecuencia, se hace necesario y relevante generar reformas educativas que respondan a los nuevos enfoques del desarrollo. La formación de maestros es relevante en la medida en que son ellos los llamados a darle sentido a la nueva lógica de la modernización de la sociedad y el Estado, fenómeno que adquiere su mayor despliegue en este período.

En este orden de ideas, el documento hace referencia a la trayectoria relacionada con la formación, capacitación y mejoramiento de los maestros rurales colombianos en el período comprendido entre 1946-1994. Este lapso, se define teniendo en cuenta las diferentes reformas educativas orientadas que determinaron particularmente la formación normalista y la normatividad de escalafonamiento del magisterio de primaria.

El texto esta divido en tres partes: La formación docente en la segunda hegemonía conservadora, que hace una relación sobre las condiciones en

Oficina de educación iberoamericana, La Educación en el Plano Internacional. Educación Primaria (Madrid: 1959), 367 
que se encontraron los maestros y maestras rurales durante este período en Colombia; el segundo hace referencia a la reformas y estructuras de las Escuelas Normales: segunda mitad del siglo XX, para ilustrar a cerca de la importancia que jugó el decreto 1955 de 1963 en el campo de la formación de los maestros rurales hasta 1974, y la reforma con el decreto 080 de 1974, el cual permite una mirada hacia los nuevos enfoque de la formación normalista, y un tercer aparte que trata sobre la formación y mejoramiento docente, donde se tiene en cuenta los aportes en este sentido desde el decreto 1955 de 1963 y el decreto 2277 de 1979 de escalafonamiento, con énfasis a los cursos-créditos y los microcentros rurales.

\section{Los maestros rurales durante segunda hegemonía conservadora}

El período 1946-1953, de cruda violencia política en Colombia, se presentó una abierta persecución a las ideas liberales por parte de los conservadores, en retaliación a lo sucedido en la década anterior por los gobiernos liberales; persecución que contribuyó al deterioro de la educación en zonas rurales del país. Esta persecución se dio en dos sentidos: el nombramiento de maestros de ideas conservadores en instituciones educativas rurales y la discriminación y eliminación física de los contrarios. La garantía de mantener maestros con algún nivel de formación y titulados en instituciones rurales se desvaneció por completo.

Una vez en el poder el nuevo régimen conservador (1946-1957), se intentó revivir el proyecto cristiano-conservador, relacionado con el confesionalismo de finales del siglo xIx y primer cuarto del siglo xx. En esta perspectiva se renovaron criterios políticos que abarcaron tanto la reforma educativa, con amplio impacto para el sector rural, como los fundamentos para la formación docente a través de las escuelas normales. Lisandro Medrano, director del Departamento Normalista del Ministerio de Educación en 1949, plantea con cierta claridad la propuesta ideológica sobre la cual debían funcionar las escuelas normales del país.

Hace referencia a la contundente oposición del gobierno conservador a los ideales expresados y practicados por el gobierno liberal: "La interpretación puramente materialista de la vida; la superestimación de los valores económicos, el determinismo histórico y geográfico, las teorías evolucionistas, labran los hondos surcos en la conciencia de los nuevos maestros. Y qué vehículo tan prodigioso de adoctrinamiento y perversión de las mentes infantiles si se dejara 
prosperar la peligrosa propaganda desde los claustros normalistas"3. Y más adelante, con criterio conservadurista y católico, continua afirmando: “[...] Queremos enseñar para algo, para cimentar las grandes verdades religiosas, las patrióticas, las sociales. Queremos que nuestros futuros maestros aprendan para algo: para vivir una vida puramente cristiana, una vida que conjugue las grandes virtudes de caridad, justicia y solidaridad"4.

El pensum normalista, hasta 1951, sufrió numerosas modificaciones: 1929, 1932, 1935, 1945, 1951. En promedio, un pensum nuevo cada cuatro o cinco años, y nadie pudo decir con suficiente conocimiento de causa que esos pensumes fueron buenos o malos, no se les dio oportunidad de ser ensayados y evaluados científicamente. El decreto 192 de 1951 se constituyó en la última versión normativa que entró a regular el pensum académico para las escuelas normales regulares ${ }^{5 *}$ de primera mitad de siglo xx. Mediante este se adopta un plan de estudios para los institutos de enseñanza pedagógica y educación normalista, el cual se extiende en un contexto teórico-práctico hasta el sexto año de secundaria.

No obstante la creación de instituciones de educación normalista y sus respectivas reformas académicas, la segunda mitad de siglo xx comienza con un magisterio precariamente formado, tanto para el nivel de primaria como de secundaria. En 1949 la nación tenía a su servicio 17.660 maestros, de los cuales solo 3.618 (20\%) eran graduados y los restantes carecían de formación normalista. Según datos de 1955, expresados por Betancur Mejía, de un total de 24.579 maestros existentes en el país, 16.728 (68,1\%) carecían de título o cualquier tipo de formación profesional o pedagógica, 748 (3,04\%) tenían título de bachiller, lo cual garantizaba alguna formación, pero ninguna pedagógica, solamente $2.747(11,17 \%)$ habían cursado estudios completos en normales elementales, y $4.356(17,3 \%)$ se habían formado en normales superiores. La conclusión es que hasta 1955, únicamente el 28,9\% del magisterio había cursado los estudios para el ejercicio de la función docente 6 un $8 \%$ más que en 1949. En este sentido, uno de los principales problemas de la educación en el período era la capacitación profesional del magisterio de primaria y secundaria en ejercicio. La formación de los maestros que

3 Lisandro Medrano, Informe del Departamento Normalista al Ministro de Educación Nacional ( Bogotá: Ministerio de Educación Nacional, 1949), 37.

Medrano, "Informe del Departamento", 37

* Hasta 1963 la educación normalista en Colombia estuvo divida en tres tipos de escuelas: Normal Superior (con 5 o 6 años), Normales Regular (con 4 años) y Normales Rurales (con 3 años).

6 Gabriel Betancurt Mejía, Documentos para la historia del planeamiento de la educación. Informe para el proyecto del primer plan quinquenal Vol. II. (Bogotá: Universidad Pedagógica Nacional, 1984), 119. 
laboraron en el sector rural a mediados del siglo XX fue realmente deplorable, como se puede apreciar en la tabla 1. En todas las cifras comparativas entre la educación rural y urbana se encuentran diferencias contundentes que afectan formidablemente la población rural; en este caso, la formación docente no es la excepción. De hecho, el porcentaje de maestros de enseñanza primaria oficial en las zonas rurales que no tenían grado llegaba al $82 \%$, mientras que este porcentaje era del $51 \%$ para las zonas urbanas, lo cual confirma la deficiente educación en todo el sector primario.

Tabla 1. Preparación de los docentes en establecimientos de primaria oficial: 1940-1950 (porcentaje de docentes no graduados)

\begin{tabular}{|c|c|c|c|}
\hline \multirow[b]{2}{*}{ AÑO } & \multicolumn{3}{|c|}{ EDUCACIÓN PRIMARIA OFICIAL } \\
\hline & TOTAL & URBANA & RURAL \\
\hline 1940 & 71,38 & 54,8 & 86,8 \\
\hline 1941 & 66,38 & 49,8 & 86,8 \\
\hline 1942 & 64,20 & 48,3 & 83,5 \\
\hline 1943 & 66,29 & 49,6 & 80,3 \\
\hline 1944 & 66,11 & 48,7 & 82,3 \\
\hline 1945 & 64,98 & 49,2 & 80,3 \\
\hline 1946 & 65,16 & 47,8 & 82,0 \\
\hline 1947 & 67,13 & 47,7 & 87,3 \\
\hline 1948 & 65,42 & 48,4 & 81,5 \\
\hline 1949 & 66,32 & 50,1 & 82,0 \\
\hline 1950 & 66,46 & 50,7 & 81,9 \\
\hline
\end{tabular}

Fuente: ICOLPE, Personal docente del nivel primario (Colombia: Serie Estadísticas básicas, 1971).

Esta situación se hace más aguda con los maestros de las zonas rurales. Las Escuelas Normales Rurales, creadas en 1934, se ocupaban de formar maestros para las escuelas rurales y, por tal medio, de prestar atención a la población campesina, que para 1951 representaba el $61.3 \%$, de 11.548 .172 de población total existente en el país 7 . Pero ninguna o casi ninguna de las maestras que salieron de estas normales trabajaron en el campo, sino en las poblaciones, por una serie de circunstancias como: la soledad a la que se veían abocadas, condiciones precarias de infraestructura y recursos escolares, la incomodidad, las grandes distancias que se debían recorrer por vías y caminos de herradura en muy mal estado, un salario más bajo en relación con las maestras y maestros urbanos y la facilidad con que los directores de educación las empleaban en el sector urbano. 
En las memorias del Ministro de Educación Rafael Azula Barrera, de 1951, se hallaron los últimos argumentos sobre los que estaba cimentada la Escuela Normal Rural, ya que posteriormente desapareció como tal: "Las Escuelas Normales Rurales se fundaron con el propósito de formar maestros para las escuelas de vereda, para campesinos y campesinas, y para que sean una fuerza poderosa que lleve su acción a todo el campesinado adulto a fin de mejorar su nivel de vida y dar una gran contribución a la transformación del país, creando las condiciones culturales mínimas y las actitudes que favorezcan la modificación gradual en los hábitos del hombre rural"s. No obstante, los objetivos perseguidos no se alcanzaron por causas como:

1. Su espíritu tomó una orientación acentuadamente académica y más bien de tipo urbano.

2. Funcionaron en grandes edificios, de dos y hasta de tres plantas, en medios típicamente urbanos [...].

3. Las actividades campesinas no encontraron su punto de apoyo [...]. Esto ocurrió, o porque las normales rurales no dispusieron de profesorado suficiente en número y en preparación o porque muchas de ellas ostentaron una importante construcción, pero sin ninguna hectárea de terreno para cultivos y actividades experimentales.

4. En su gran mayoría, los maestros egresados de tales establecimientos buscaron otras ocupaciones o sirvieron a las escuelas urbanas.

Las escuelas normales, continúan con la división existente desde 1934: escuelas normales rurales, escuelas normales regulares, públicas y privadas, y dividas por sexos. En los años de 1952 a 1957 se cierra la Escuela Normal Superior por decreto de Laureano Gómez y su ministro de educación, quienes consideraban que no era aceptable, desde el punto de vista moral, el que los alumnos de uno u otro sexo convivieran y trabajaran juntos en las mismas aulas, y en virtud de ello determinaron bifurcar la Facultad-Madre de Ciencias de la Educación, enviando a las mujeres al Instituto Pedagógico de la avenida Chile de Bogotá y a los varones a la Facultad de Tunja.

Rafael Azula Barrera, Memorias del Ministro de Educación Nacional (Bogotá: Ministerio de Educación Nacional, Departamento de Enseñanza Normalista, 1951), 118. 
Tabla 2. Alumnos matriculados en Escuelas Normales del país: 1949

\begin{tabular}{|cccc|}
\hline Normales Nacionales & Matriculados & Becados & $\begin{array}{c}\text { Último } \\
\text { año }\end{array}$ \\
\hline Alumnos matriculados en normales regulares & 1.211 & 622 & 159 \\
Alumnos matriculados en normales rurales & 45 & 45 & 14 \\
TOTAL & 1.256 & 667 & 173 \\
Alumnas matriculadas en las normales regulares & 1.385 & 586 & 134 \\
Alumnas matriculadas en las normales rurales & 760 & 457 & 194 \\
TOTAL & 2.147 & 1.133 & 328 \\
Normales Departamentales* & & & \\
TOTAL & Matriculados & Becados & Último \\
& 229 & & año \\
Plumnos matriculados en normales regulares de varones & 185 & & 34 \\
Alumnos matriculados en normales rurales de varones & 414 & & 34 \\
Privados & & & Último \\
año \\
Alumnos matriculados en planteles regulares de varones & 124 & & 5 \\
Alumnas matriculadas en planteles regulares de señoritas & 717 & & 78 \\
Alumnas matriculadas en normales rurales de señoritas & 595 & & 88 \\
TOTALES GENERALES & $\mathbf{6 . 3 8 9}$ & $\mathbf{1 . 8 0 1}$ & $\mathbf{8 2 2}$ \\
\hline
\end{tabular}

Fuente. Ministerio de Educación Nacional, Memorias del Ministro de Educación Nacional (Eliseo Arango) 1949.

* No se encontraron datos de alumnas matriculadas en Normales Rurales

En la tabla 2 se puede observar y analizar que a pesar de que existía un considerable número de escuelas normales, dispersas a lo largo y ancho del territorio nacional, el número de estudiantes no corresponde a las necesidades educativas, tanto en formación como en ejercicio. Así también, permite considerar que el número de mujeres que se matriculaban y permanecían en normales regulares y rurales, nacionales, departamentales y privadas, era mayor que el de los varones, sin embargo, el número de varones becados por el Estado era más alto que el de mujeres. La deserción de varones y mujeres que ingresaban a las escuelas normales regulares era más alta que la de las escuelas normales rurales; esta situación se explica en la medida en que la escolaridad para las normales rurales sólo alcanzaba hasta el tercer año de secundaria y su vinculación laboral no era diferente a los egresados de otras normales, mientras quienes ingresaban a las normales regulares debían permanecer hasta el quinto o sexto año. No obstante, el ingreso de estudiantes a las escuelas normales rurales era inferior al de las instituciones regulares. 


\section{Reformas y estructuras de las Escuelas Normales: segunda mitad del siglo XX}

Con el Primer Plan Quinquenal de Educación se inició en Colombia un período de transformación educativa en todo sentido, influenciado por las políticas internacionales de modernización y desarrollo económico y cultural. Las reformas a la educación determinaron la formación docente. Martínez Boom asocia las décadas de los años sesenta hasta los ochenta como el período de la introducción de la escuela expansiva: "La escuela se presentó como la gran alternativa para la erradicación de la ignorancia, el analfabetismo y, en general, el atraso social, con lo cual se inauguró un período de crecimiento escolar sin precedentes en la historia educativa latinoamericana"9 [y colombiana].

En 1962, una comisión conjunta de la Unesco, el Banco Internacional de Reconstrucción y Fomento -BIRF- y la Agencia Internacional de Desarrollo de los Estados Unidos formuló algunas recomendaciones básicas sobre la elaboración de un plan integral de desarrollo educativo. Acogiendo sus sugerencias y adoptando sus recomendaciones concretas, el Gobierno Nacional reestructuró la Oficina de Planeamiento y con ella como base institucional creó la Misión de Planeamiento Integral de la Educación que, coordinada por un experto nacional y con la colaboración de funcionarios de las agencias internacionales, se encargó de la formulación de un plan educativo integral para Colombia. El Plan Integral de Educación, que surgió como parte del Plan de Desarrollo Económico y Social de 1961, se propuso como líneas generales reformar la educación elemental, normalista y media. De esta manera, entran en juego las ideas liberales de Occidente; claramente expresado por el Ministro de Educación Pedro Gómez Valderrama:

El concepto politico de democratización de la cultural, la elevación de la educación a la categoría de derecho bumano fundamental irrenunciable, la inevitable acción reguladora o intervencionista del Estado, en una sociedad cada vez más compleja, los fenómenos de explosión demográfica, la comprobación de que la educación es por si misma un factor de mejoramiento de la productividad, lo que ha becho que se le considere como inversión rentable a largo plazo, o sea una especie de bien de producción y de consumo simultáneamente; la rapidez. con que se operan los cambios en la vida contemporánea, la misma pequeñez relativa del mundo debido al prodigio de las comunicaciones, son otras de muchas razones que obligan a encarar los problemas educativos con criterio reformista o por lo menos revisionista ${ }^{10}$.

\footnotetext{
9 A. Martínez Boom, De la escuela expansiva a la escuela competitiva. Dos modos de modernización en América Latina (Barcelona: Anthropos, 2004), 4.

10 Pedro Gómez Valderrama, Memorias del Ministro de Educación Nacional. Tomo I (Bogotá: Ministerio de Educación Nacional, 1964), 16.
} 
Los albores de las primeras reformas normalistas de la segunda mitad del siglo xx se inauguran con el decreto 2617 de 1959, con el cual se propone reorganizar el sistema de enseñanza normalista y se promueve la formación de maestros. Con esta norma se pone fin a la diferencia que existe entre las escuelas normales regulares y escuelas normales rurales. Se pretende con ello generar las primeras alternativas hacia la puesta en marcha del Plan de Extensión de Educación Primaria.

Los planteles llamados a cumplir con este cometido fueron las escuelas normales superiores. Las restantes escuelas normales quedaron gradualmente transformadas a partir de 1960 en planteles del primer ciclo (cuatro primeros años) de enseñanza secundaria [...]. La formación del magisterio se concertaría en el segundo ciclo de enseñanza secundaria, quinto y sexto años, con materias profesionales necesarias para la formación docente. El título que optarían los futuros egresados sería el de "Bachiller Normalista"11. Aquí se da el primer paso para el rompimiento de una estructura académica, pedagógica y cultural que había marcado el hito en la formación de maestros en Colombia.

Con el decreto 1710 de 1963, se adopta el plan de estudios de la educación primaria colombiana- situación que hace indispensable la revisión de los métodos de formación de maestros, vale decir, de la educación normalista, como presupuesto indispensable para el mejoramiento cualitativo de la escuela primaria. Con el propósito de ajustar las características del maestro a las nuevas exigencias planteadas por las reformas que operaban sobre la escuela primaria (decreto 1710 de 1963), se aprueba el decreto 1955 de 1963, que reorganiza la educación normalista, acogiéndose a los planteamientos centrales del Primer Plan Quinquenal de Educación.

El decreto 1955 de 1963 se constituyó en la mayor transformación que sobre formación de maestros de primaria se realizara en el país hacia la segunda mitad del siglo XX. Con la reforma de las escuelas normales se pretendió ajustar la formación docente a los planes y programas educativos (decretos 45 de 1962 y 1710 de 1963), así, entonces, la reforma educativa tuvo como propósito entrar a los procesos de modernización. Es decir, la formación de maestros requería ser adaptada al progreso de las ciencias y la cultura, y al desarrollo general de país. Un primer paso es unificar las instituciones de formación de maestros, el decreto expone que: "Todo plantel educativo que se dedique a la formación de maestros [...] se denominará

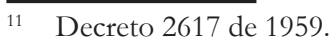


Escuela Normal, a la cual se le indicará si es de varones o de señoritas"12. En ese mismo orden, en el artículo cuarto define las funciones: a) Formar maestros idóneos para las escuelas primarias del país; b) Organizar servicios de capacitación profesional para los maestros en ejercicio que carecen de título correspondiente; c) Organizar servicios de perfeccionamiento para maestros titulados; d) Extender su acción social, cultural, técnica a individuos y grupos de la comunidad"13.

Esta reforma de las escuelas normales se extiende a la estructura, finalidades, organización técnica y administrativa, a las escuelas primarias de aplicación, demostrativas y de experimentación, a los planes y programas de estudio, régimen salarial de los maestros y calendario escolar, evaluación y promoción de estudiantes. Constituido así el decreto 1955 de 1963, desempeñó papel preponderante en la división del trabajo entre el campo administrativo y el campo pedagógico y garantizó un discurso cuyas prácticas pedagógicas estuvieron orientadas a mantener el orden institucional a través de los procesos de planeación. En este sentido, lo más importante en la formación del maestro era desarrollar habilidades concretas para la planeación: saber planear una clase, utilizar el material didáctico, formular objetivos y saber evaluar. La reforma se queda en el papel del maestro formador para una educación instrumental, incluido en un proceso híbrido entre lo tradicional y lo moderno, sin modernidad, pero que cumple cabalmente los planes y programas emanados del Ministerio de Educación, no importa si responden o no a las necesidades del sector donde se halla inmersa la institución.

De otro lado, las reformas a la educación primaria y a las escuelas normales entre sí fueron inconexas. Mientras que los programas establecidos para la educación primaria (decreto 1710 de 1963) hacían énfasis en la pedagogía centrada en el alumno (a través del método de problemas, de las prácticas de descubrimiento, participación y comunicación), los programas de la normal continuaban con una pedagogía centrada en el maestro, quien, siguiendo las "indicaciones didácticas generales", debía enseñar, capacitar y realizar las técnicas apropiadas conforme a lo establecido por los programas oficiales.

El decreto 1955 reprodujo el lenguaje de los nuevos requerimientos sociales y educativos, expresados por los organismos y agencias internacionales: expansión acelerada del nivel de primaria y la tendencia a la democratización del nivel secundario, y planteó a las escuelas normales una triple función: formación, capacitación y mejoramiento educativo.

$\begin{array}{ll}12 & \text { Decreto } 1955 \text { de } 1963 . \\ 13 & \text { Decreto } 1955 \text { de } 1963 .\end{array}$ 
Con la reforma a las escuelas normales se unifica el sistema de formación docente, es decir, las escuelas normales rurales, las escuelas normales regulares y la escuela normal superior, y pasa a tener un fin único en los procesos de enseñanza, tanto en las áreas rurales como urbanas: "se formará un solo tipo de maestro para la enseñanza primaria, con preparación adecuada para actuar eficientemente en el medio urbano o rural y para adaptarse a las peculiares condiciones regionales o locales" 14 .

Reorganizadas de esta manera la educación primaria y secundaria, por un lado, y la formación docente a través de las normales, por otro, cuyo énfasis pedagógico, didáctico y metodológico estuvo orientado para la educación urbana, las escuelas rurales y sus maestros se vieron abocados a continuar con el régimen tradicional que los había caracterizado desde principios de siglo XX. Los planes, programas y escuelas normales unificadas requerían de una plataforma de recursos técnicos, humanos y financieros que colmara de alguna manera las necesidades educativas del momento, de la cual careció en buena parte el sector rural.

El decreto 080 de 1974 proporciona nuevos elementos para el análisis de las escuelas normales. Si bien no se trata de una reforma propiamente dicha sobre este tipo de instituciones, incluye la formación pedagógica en el currículo de la educación media. Este decreto regula la formación de maestros desde las perspectivas temáticas, ideológicas, teóricas y prácticas oficiales. Es así como estableció un plan fundamental mínimo de estudios, en consonancia con las modernas tendencias educativas y las necesidades del país. Las tendencias educativas a las que se refiere el decreto 080 de 1974 estaban relacionadas con la tecnología educativa y el marco del diseño instruccional, que para las escuelas normales es adaptado a través de la resolución 4785 de 1974, con la cual se reglamenta la formación normalista.

La resolución 4785 de 1974 establece un currículo no especializado en áreas del conocimiento con sus correspondientes números de materias pedagógicas, con énfasis en una cultura humanista sobre la cultura pedagógica o profesional y sobre el área de habilidades y destrezas vocacionales. Esta reglamentación fortaleció el proceso educativo instrumental, y, mediante la vocacionalización, pretendió abrir el paso a los estudiantes a la educación superior o el trabajo, pero no estuvo acorde con las condiciones sociales y de empleo del país. 
De la misma manera, la resolución 4785 de 1974 estipuló la organización y administración del currículo a través de la planeación: objetivos precisos, contenidos, recursos, evaluación en función de conductas observables, aspectos que no estuvieron basados en ninguna teoría específica de aprendizaje. Se refuerza el modelo tradicional, porque en ningún momento entró en contradicción con los contenidos y presupuestos oficiales expuestos, tampoco propuso recreación de contextos para el acceso al conocimiento. No hubo una transformación del modelo pedagógico en sus esquemas y estructuras tradicionales. Ubicaba al alumno en el "centro del aprendizaje" y al maestro como "guía y orientador de las tareas educativas". "Se trata de una transmisión parcelada de saberes técnicos mediante un adiestramiento experimental que utiliza la tecnología educativa.

Con el decreto 080 y la resolución 4785 de 1974, las escuelas normales pierden su identidad, ya que establecen un plan mínimo para todo el bachillerato y dan a los estudios normalistas el carácter de bachiller pedagógico, y no el de maestro.

Eldecreto 088 de 1976 establece como función (artículo 32) de la Dirección General de Capacitación y Perfeccionamiento Docente, Curricular y Medios Educativos precisar los currículos, los objetivos particulares, los contenidos y los métodos que corresponden al medio rural y urbano en las distintas regiones del país. Dos años más tarde, el decreto 1419 de 1978 establece los marcos legales para el diseño y la administración curricular. Con el propósito de establecer los fines del sistema educativo, entiende por currículo el conjunto planeado y organizado de actividades, en el que participan alumnos, maestros y comunidad para el logro de los fines y objetivos de la educación.

El decreto 1419 de 1978 no presenta reformas a fondo para las escuelas normales. Díaz y Chávez afirman que este decreto "se queda en la retórica oficial: mejoramiento cualitativo, ampliación de servicios educativos y adaptación al desarrollo del país. La flexibilidad, como mecanismo de acción pedagógica, adquiere carácter renovador" ${ }^{\prime 1}$. Si bien el decreto introduce el concepto de flexibilidad curricular, presenta un modelo riguroso sobre el cual se debe planear y organizar el proceso educativo. En el artículo 10 del decreto 1419, la formación normalista queda sustituida por una de las modalidades de la "Educación Media Vocacional", que conduce al Bachillerato en Tecnología en modalidad pedagógica. Con este cambio, que no es una simple sustitución de palabra, culmina el proceso de disolución de toda una tradición.

15 M Díaz V. y Ca Chávez B, "Las reformas de las escuelas normales un área de conflicto", Revista Educación y Cultura No 20 (1990): 20. 
Las reformas muestran las transformaciones sufridas por las instituciones educativas en el paso de la primera a la segunda mitad de siglo. Desde la perspectiva pedagógica, se pasa de una propuesta activa, bajo el dominio de ideas liberales y laicistas, que impulsan un proyecto de modernización de la educación, y que va hasta 1946, cuando el régimen conservador asume nuevamente el poder, a una que reorienta la educación normalista en la búsqueda de ponerla de nuevo al servicio del catolicismo, la patria y la formación de "buenos ciudadanos".

De 1958 a 1994 la formación docente a través de la escuela normal se ve influenciada ya no por los debates pedagógicos de la escuela activa o tradicional, laica o clerical, sino por los criterios economicista y productivos del nuevo orden, que ponen al centro la expansión educativa (la universalización de la escuela primaria) y el saber hacer. En este sentido, la tecnología educativa instrumental de la educación retoma un proceso que alcanza el tercer milenio.

Las reformas a las escuelas normales revelan los procesos e intentos de acomodamiento a los continuos cambios que los ámbitos: económico, sociocultural y político implicaron a la sociedad y determinaron el lugar de Colombia en el resto del mundo. $\mathrm{Al}$ referirse $\mathrm{Helg}^{16}$ a los maestros rurales de primera mitad de siglo XX expresó que si bien "los maestros no tenían una formación, ni títulos como maestro, sí seguían al pie de la letra los programas oficiales". Esta situación no cambió hacia la segunda mitad del siglo, por el contrario, las reformas en la formación docente marcaron pautas hacia un proceso de planificación rígida, resultado de modelos pedagógicos de transmisionismo conductista; rigidez que se afirma tanto en el aprendizaje de los estudiantes - centro de la reforma-como en las condiciones formativas y culturales del maestro.

Las reformas a las escuelas normales fueron consecuentes con los discursos pedagógicos, metodológicos y administrativos, fuentes de poder y dominio oficialista, en la perspectiva de los organismos internacionales, con claros efectos sobre los sujetos, las instituciones y las prácticas educativas. Tal como lo plantea Foucault: "El poder es algo que opera a través del discurso, puesto que el discurso mismo es un elemento dispositivo estratégico de relaciones de poder" ${ }^{17}$. En este caso el discurso son las reformas mediadas por leyes, decretos y resoluciones: "acontecimientos políticos a través de los cuales el poder se transmite y se orienta"18.

\footnotetext{
16 A. Helg, La Educación en Colombia: 1918-1957 (Bogotá: Universidad Pedagógica Nacional, 1987).

17 Michel Foucault, "Diálogo sobre el discurso", en Estética, ética y hermenéutica, eds. Michel Foucault, Decreto 2617 de 1959 (Barcelona: Paidos, 1999), 59.

18 Foucault, "Diálogo", 59.
} 
Para finalizar el período histórico en estudio (1946-1994), recurrimos a la última reforma que se hizo a las escuelas normales. Cerrando la década de los años ochenta, la escuela normal presenta crisis en sus fines, competencias y demanda. De un pasado de reconocidos méritos se llegó a un momento en que se cuestionó su existencia. Perdió terreno en el contexto del sistema educativo. Se debatió entre escuela normal o colegio de bachillerato. De otro lado, tuvo que enfrentar la competencia de la educación superior, a través de las licenciaturas en educación primaria. En este período se presentó una amplia oferta de formación en educación en universidades públicas y privadas: presenciales, semipresenciales y a distancia.

De otro lado, los cambios culturales, sociales y económicos de los últimos tiempos cuestionaron el papel de las escuelas normales y la formación de los bachilleres pedagógicos, especialmente en el tipo de maestro que se venía formando. Las nuevas tendencia hacia un discurso centrado en el desarrollo humano, los medios de comunicación, los progresos del conocimiento sobre la niñez y el desarrollo del cerebro, la informática y el progreso en ciencia y tecnología exigieron nuevas estrategias hacia la formación de maestros orientados hacia procesos visionarios de la educación para el desarrollo.

El Ministerio de Educación Nacional se da a la tarea de realizar un autodiagnóstico, a través de un formulario entregado a las diferentes escuelas normales del país. Previamente se había convocado a las Facultades de Educación y al Centro de Estudios e Investigaciones Docentes de la Federación Colombiana de Educadores (Fecode) para consultarlos al respecto; no obstante, los aportes de estas entidades no fueron tenidos en cuenta para la elaboración del proyecto de ley 114 de 1989. Por el contrario, se retoman los resultados del diagnóstico y la evaluación hecha por Kurmen y Briones, publicada en $1978^{19}$, donde encontraron que el número de establecimientos que impartía formación pedagógica ascendía en 1977 a 209, de los cuales el $78 \%$ eran oficiales y el 22\% privados. Esta situación llevó a que se hicieran las siguientes recomendaciones: que no se permitiera la creación de ninguna escuela normal, al tiempo que indica la necesidad de hacer una depuración de las normales existentes, transformando las que no llenan los requisitos en planteles de otras modalidades distintas; que la educación normalista se impartiera a nivel de carrera intermedia y que se le dé nuevamente a sus establecimientos el nombre de escuelas normales (este había sido cambiado a partir del decreto 080 de 1974), y se otorgará a su vez el título de maestro, que por el mismo decreto había sido cambiado por el de bachiller pedagógico.

\footnotetext{
19 Alcira Kurmen Rojas y Guillermo Briones, Evaluación de las Escuelas Normales de Colombia Vol.1 (Bogotá: MEN-PNUD-UNESCO, 1978), 25; Alcira Kurmen Rojas y Guillermo Briones, Evaluación de las Escuelas Normales de Colombia Vol .3 (Bogotá: MEN-PNUD-UNESCO, 1978), 40.
} 
Bajo estos fundamentos, el Ministerio de Educación presentó al Congreso de la República el proyecto de ley 114 de 1989, a partir de los siguientes fundamentos: a) Ampliación del nivel de Educación Media Vocacional en dos años - grados doce y trece- en jornada completa ordinaria; b) Expedición del título de maestro al finalizar y aprobar el grado trece, con opciones de énfasis en su formación; y c) Dedicación exclusiva de las escuelas normales a la formación de maestros.

No obstante las apreciaciones y argumentos de rechazo, que la comunidad académica hiciera al proyecto de ley 114 de 1989, el Congreso de la República aprobó uno nuevo, el decreto 1348 de 1990, donde se retoma en su totalidad la iniciativa de dicho proyecto de ley. Con este decreto se reforman las escuelas normales y se adopta un sistema especial de formación de maestros, considerando que la última reforma para estas instituciones se había hecho con el decreto 080 de 1974. Las escuelas normales quedan supeditadas a un régimen especial: ocho años de formación, vuelven a expedir el título de maestro, le otorgan dedicación exclusiva a la formación de maestros y dan la posibilidad a sus egresados de continuar estudios superiores.

Planteada así la reforma a las escuelas normales, con el decreto 1348 de 1990, se abren las expectativas de un proyecto remodernizador de la educación primaria. Dos ingredientes determinan los nuevos criterios formativos: la necesidad de responder a la construcción del conocimiento, un maestro que avance hacia los esquemas de la creación e innovación del saber, en virtud de lo cual debe ser investigador, y el maestro animador del desarrollo comunitario, discurso que se mueve en el interior del modelo neoliberal.

\section{Capacitación y perfeccionamiento docente}

La segunda mitad del siglo XX es época de grandes transformaciones económicas, políticas, culturales, científicas y tecnológicas. La educación empieza a tener una profunda relevancia como eje dinamizador de estas transformaciones. En consecuencia, las reformas educativas y la implementación de la Escuela Nueva para las escuelas rurales debieron tener en cuenta tanto la formación del maestro a través de las escuelas normales y las Facultades de Educación, como los procesos de capacitación y mejoramiento docente.

Con el decreto 1955 de 1963 se les asigna a las escuelas normales el servicio de capacitación y perfeccionamiento profesional. De esta manera, al servicio de la capacitación profesional tenían acceso "los maestros en servicio que carecían del título que los acreditara como tales o quienes poseían título de 
maestro rural o maestro elemental; y al servicio de perfeccionamiento, quienes acreditaban el título de Institutor o Maestro Superior o quienes acreditaran el título de Maestro rural o el certificado de Maestro elemental" ${ }^{20}$. Además, con el decreto 1955 se pretendió buscar una solución al gran problema educativo del momento: la ausencia de una masa de maestros formados acorde con las exigencias educativas (universalización de la educación primaria en los fundamentos básicos y educación secundaria para la productividad), que a la vez deberían orientar procesos sociales y económicos enfocados a dar respuesta al nuevo orden social y económico impulsado por las teorías del desarrollo y los nuevos procesos de modernización. En esta circunstancia, la formación y capacitación docente se constituye en una necesidad prioritaria no sólo en Colombia, sino también en América Latina.

Los diagnósticos económicos y sociales, Currie, Lebret y la Tercera Misión Alemana dejan al descubierto grandes problemas de la educación primaria. El aspecto más preocupante que se encontró en la década de los años sesenta fue tal vez el de la calidad de la educación primaria, manifiesta en una baja asimilación de conocimientos por parte de los estudiantes y altos índices de deserción y repitencia. Parte de la raíz del problema se centraba en la falta de preparación académica y metodológica del magisterio. Una investigación diagnóstica realizada en 1966 por quien más tarde sería el director de la Misión Pedagógica Alemana confirma esta hipótesis: "los alumnos sin diferencias regionales ni sociales, no estaban adquiriendo los conocimientos básicos que se suponía debería impartirles la escuela, y los maestros a su vez no poseían los conocimientos necesarios para implementar la reforma de $1963^{\prime 21}$.

Las políticas educativas para el mejoramiento docente de mediados de la década de los años sesenta se centraron en dos instrumentos básicos: la capacitación para docentes en servicio y el suministro de materiales didácticos para ser utilizados en el proceso de enseñanza-aprendizaje. Bajo estos presupuestos se firma un convenio bilateral con el gobierno alemán para "el desarrollo de la enseñanza primaria en la República de Colombia mediante reforma en los sectores de perfeccionamiento del profesorado, de la organización práctica de la enseñanza y de los medios de enseñanza" (artículo primero) $)^{22}$. Se trataba de prestar ayuda para desarrollar los programas establecidos de acuerdo con el decreto 1710 de 1963. Esta ayuda se efectuó a

\footnotetext{
$20 \quad$ Decreto 1955 de 1963.

21 Walter Kaessmann, "Diagnóstico sobre la situación actual de la Enseñanza de Matemáticas en la Escuela Primaria Colombiana", en Análisis de una experiencia, eds. María Cristina Rojas de Ferro (Bogotá: Universidad Pedagógica Nacional, 1966), 4.

22 Rojas, "Análisis", 5.
} 
través de tres actividades principales: a) elaboración de guías para el desarrollo de los nuevos planes de estudio; b) elaboración de material didáctico para auxiliar al maestro en su práctica de enseñanza, y c) capacitación a los maestros en el uso de las guías y el material didáctico elaborado ${ }^{23}$.

La capacitación para el manejo de las Guías Alemanas constituyó el único programa de perfeccionamiento para los maestros de primaria activos de 1968 a 1978 en Colombia.

\section{Los cursos-crédito y los microcentros rurales}

La capacitación y el mejoramiento docente como política nacional de 1979 a 1994 se establece bajo dos estrategias: los cursos-crédito para ascenso en el escalafón docente y los microcentros.

Los cursos-crédito, eran cursos presenciales que otorgaban créditos para ascenso en el escalafón (decreto 2277 de 1979, mediante el cual se adoptan normas para el ejercicio de la profesión docente); eran aprobados y administrados por los Centros Experimentales Piloto y las Secretarías de Educación de cada uno de los departamentos. Con el decreto 2277 de 1979 se unifica el escalafón docente de primaria y secundaria, urbano y rural, por creación y grados; "se entiende por Escalafón Docente el sistema de clasificación de los educadores de acuerdo con su preparación académica, experiencia docente y méritos reconocidos" la clasificación de los educadores, según el decreto, estaba constituido por catorce grados en orden ascendentes, del grado primero hasta el decimocuarto.

El decreto 2277 de 1979 estipuló dentro de las reglas especiales para ascenso en el escalafón: el tiempo de servicio, ascenso por título docente y cursos de capacitación. En este sentido la capacitación y el mejoramiento del docente como requisitos de ascenso al escalafón se constituyeron en política nacional. "Los cursos de capacitación y actualización que realice el educador para ascenso serán tenidos en cuenta como crédito para obtener el título de bachiller pedagógico, licenciado en ciencias de la educación u otros, en las condiciones que determine el reglamento ejecutivo"24.

El artículo quinto del decreto 2277 de 1979 estableció que sólo podrán ser nombrados para ejercer la docencia en planteles oficiales quienes posean

23 Martínez et al., Reformas de la Enseñanza en Colombia: 1960-1980. Del énfasis didáctico al énfasis curricular, Revista Educación y Cultura No. 15 (1988): 12-21.

24 Ministerio de Educación Nacional. Decreto 2277 de 1979, artículo 13. 
título de docente o acrediten estar inscritos en el Escalafón docente. Sin embargo, establece un parágrafo con las siguientes excepciones:

En las zonas rurales de difícil acceso y poblaciones apartadas a que se refiere el artículo 37 o para educación especial, podrán nombrase para ejercer la docencia en los niveles preescolar, básica primaria y básica secundaria, personas que acrediten titulo de bacbiller en cualquier modalidad, siempre y cuando no exista personal titulado o en formación que esté en capacidad de prestar el servicio requerido $[\ldots]^{25}$.

El parágrafo que estipula el artículo quinto del decreto 2277 de 1979 es contradictorio con lo que la norma plantea en términos generales: "las personas $[\ldots]$ sólo podrán ingresar al escalafón cuando adquieran título docente en programas regulares o a través de la profesionalización de que trata el artículo $57^{\prime 26}$. Frente a este aspecto se puede afirmar que ser maestro rural y no haber ingresado al escalafón docente era una situación que ponía en riesgo la estabilidad laboral y salarial del maestro, lo mismo que la posibilidad de que en las zonas rurales se obtuvieran maestros calificados con formación pedagógica.

Bajo la presión del escalafonamiento docente, única forma de salvaguardar la condición laboral, los maestros de escuelas rurales se vieron obligados a formarse en áreas de la educación, ya fuera validando la normal, a través de formación de programas de licenciatura a distancia, especialmente las relacionadas con educación básica primaria, o a través de cursos de profesionalización. Como estrategia para solventar la crisis de formación de maestros de áreas apartadas se dio una proliferación de programas a distancia en universidades públicas y privadas, y en este mismo sentido, así como en las décadas de los años cincuenta y sesenta se dio la necesidad de titular docentes para las escuelas rurales, se produce el giro hacia la profesionalización.

Los microcentros rurales, se presentan como una estrategia de capacitación que valorando la experiencia y vivencia de los docentes los involucra en procesos de autoformación maestro a maestro, intercambiando conceptos y compartiendo actividades para buscar soluciones a problemas de aula o de relación con la comunidad. Inicialmente el Microcentro fue concebido como

\footnotetext{
Ministerio de Educación Nacional. Decreto 2277 de 1979, artículo 13.

26 Decreto 2277 del artículo 57 de 1979 trata de los objetivos: "La capacitación docente cumplirá los siguientes objetivos: a) Profesionalización a los educadores sin título docente, que se encuentren en servicio; b) Actualización a los educadores sobre los adelantos pedagógicos, científicos y tecnológicos; c) Actualizar a los educadores en las técnicas de administración, supervisión, planeamiento y legislación educativa; d) Especializar a los educadores dentro de su propia área de conocimientos; e) Proporcionar a los educadores oportunidades de mejoramiento profesional mediante los ascensos en el escalafón".
} 
un mecanismo para impulsar el conocimiento y aplicación de la Renovación Curricular ${ }^{27}$ en las zonas urbanas. En las áreas rurales, a través del programa Escuela Nueva, se da paso a la innovación y la experimentación para la formación y capacitación de los maestros; esta propuesta se plasma a través de los Microcentros Rurales, alrededor de una escuela demostrativa.

Los Microcentros Rurales se constituyen en una opción de "experiencia pedagógica" que demostraba la importancia de la agrupación de los docentes para apropiarse de su proceso de capacitación y de formación permanente, así como de una reflexión que permitió detectar las necesidades y acordar el enfoque, la importancia y la perspectiva de la capacitación y de la investigación para la definición de los problemas y el desarrollo de las soluciones respectivas.

La propuesta de los Microcentros, como opción de capacitación permanente para los docentes, fue animada por el Ministerio de Educación Nacional de Colombia a partir de 1985. En la escuela rural, la propuesta fue la de agruparse en una escuela demostrativa, escogida por los docentes a partir de una serie de criterios, entre los cuales estaba el que los docentes fueran dinámicos, líderes, animadores e innovadores de procesos pedagógicos y educativos, y que la escuela propuesta estuviera ubicada en un encuentro de caminos vecinales, donde confluyeran desde el punto de vista geográfico varias de las escuelas participantes. Los Microcentros Rurales son definidos como verdaderos círculos de calidad para el desarrollo y mejoramiento de la Escuela Nueva, teniendo en cuenta los problemas de cada escuela y de cada localidad; estaban conformados por los maestros de las escuelas vecinas que se reunían una vez al mes para analizar los problemas y discutir resultados. Los Microcentros fueron el espacio institucionalizado desde donde se estructuraron los esfuerzos de las escuelas, se reorganizaron y diseñaron currículos para que respondieran a las necesidades de los alumnos y la comunidad y para que sirvieran como orientadores de las políticas educativas a nivel local.

La metodología utilizada para el trabajo pedagógico en los Microcentros rurales era la del taller permanente. La Escuela Demostrativa escogida fortalecía la propuesta del Programa Escuela Nueva, al mismo tiempo que las escuelas cercanas trabajaban alrededor de esta modalidad. Cada escuela adoptaba el modelo de acuerdo con las necesidades de la comunidad y su condición cultural, cuyas experiencias contribuían a fortalecer el programa, no a replicarlo.

\footnotetext{
$\overline{27 \quad \text { Decreto } 2277,4 .}$
} 
El programa Escuela Nueva y su proceso de capacitación docente a través de los Microcentros rurales ocuparon un lugar central de innovación pedagógica y educativa para áreas rurales colombianas, con resultados positivos en el desarrollo cognitivo, social y comunitario. Rosa María Torres afirma al respecto: "La formación docente enfatiza la dimensión pedagógica, así como la motivación y la capacidad para innovar. Los microcentros reúnen en su concepción, de hecho, una serie de cuestiones consideradas claves en toda estrategia de formación docente: la necesidad de una formación continua y en servicio, basada en el contacto y el trabajo grupal entre docentes, el intercambio de experiencia y el análisis crítico de la propia pedagogía"28.

A pesardequelo Microcentros rurales fueronuna estrategiade capacitación, planeación e interacción docente en la totalidad de los departamentos -con excepción de Caldas y Huila-, para la mayoría de instituciones y maestros no fue una experiencia significativa. En este sentido es posible comparar el desarrollo del programa Escuela Nueva entre departamentos: en Caldas, por ejemplo, se encuentra una experiencia exitosa, que radicó en el hecho de que la capacitación docente e implementación de recursos escolares estuvo a cargo del Comité de la Federación Colombiana de Cafeteros, cuyo propósito fue el de vincular la educación rural al progreso y desarrollo del café.

\section{CONCLUSIÓN}

El período de la Segunda Hegemonía Conservadora, se constituyó en uno de los más críticos para los maestros rurales colombianos, en lo que respecta a la formación, práctica pedagógica y estabilidad laboral. Con el recrudecimiento de la violencia política entre los partidos liberal y conservador, que se arrecia en los campos colombianos, los maestros y maestras y la escuela rural, se ven afectados directamente: persecución política, particularmente hacia aquellos con ideas liberales, nombramiento de maestros sin ningún tipo de formación académica ni pedagógica, suspensión de cargos, muchos fueron asesinados por el bando contrario, otros desplazados de manera violenta. A ésto se sumó, el interés del gobierno conservador por rescatar las viejas y refundidas propuestas pedagógicas tradicionales centradas en la moral y la religión católica de primera mitad de siglo. La formación del maestro durante este período, fue nula, a pesar de las diferentes reformas efectuadas a la educación normalista.

Con el decreto 1955 de 1963 se reforma de manera contundente la educación normalista, buscó unificar la formación del maestros en todos

28 Rosa María Torres, "Alternativas dentro de la educación formal: El programa Escuela Nueva en Colombia” Revista Colombiana de Educación No 32, (1996). 
sus ámbitos. El propósito era responder al orden mundial fundado en el discurso del desarrollo y las nuevas tendencias tecnológicas. Establece los indicadores para la formación y capacitación docentes. Bajo estos parámetros, se contrata una comisión alemana (es la tercera), encargada de capacitar a los maestros rurales y urbanos para asumir los requerimientos establecidos por los Decretos 45 de 1962 y 1710 de 1963, sobre reforma a la educación básica.

En este orden deideas, se diseñan las Guías Alemanas que se constituyeron en el único material didáctico con el cual contaron los maestros rurales para el ejercicio de la docencia en el medio. Estas guías se utilizaron, desde 1967 hasta 1975 como único material didáctico. En algunas escuelas rurales del país sólo a partir de 1975, se dio un cambio pedagógico y didáctico con la propuesta de la escuela nueva.

Con la reforma a la educación básica (decretos 45de 1962 y1710 de 1963) y la reforma a la educación normalista (decreto 1955 de 1963), se establecen los derroteros para el establecimiento de una educación para el desarrollo económico. En esta perspectiva entran en juego intereses de organismos internacionales y nacionales. Se crean programas desde las instancias administrativas del Estado Colombiano, que si bien, contribuyen a la expansión de la educación básica y universitaria, no ocurre lo mismo con la calidad educativa, ni particularmente con la educación rural, la cual es inequitativa con relación a la educación para la población urbana. Por el contrario, la formación de los maestros [rurales], se constituyó en un proceso lento y penoso, que solamente logra cierto posicionamiento a partir de 1975, a través de la implementación del programa Escuela Nueva.

Con el decreto 2277 de 1979, se inicia una nueva era en la formación y perfeccionamiento docente. Se unifica el escalafón docente, y se establece un sistema salarial equivalente para los maestros rurales y urbanos y de educación básica y media. A través de este medio, los maestros rurales se ven abocados a la formación profesional y la capacitación como único medio de ascenso al escalafón. Sin embargo, la práctica de las políticas oficiales, no correspondieron, como era debido, a la formación, capacitación y mejoramiento docente. El sistema de créditos y los microcentros se vieron afectados por intereses netamente económicos, dejando de lado la única oportunidad para preparar adecuadamente un magisterio que respondiera realmente a los problemas educativos del país y más aún a los sectores rurales.

Un aspecto importante de destacar en la historia de la educación rural colombiana de segunda mitad de siglo XX está justamente relacionada con 
los maestros, tanto en el ámbito de la formación y capacitación como en el papel protagónico que representaron en el proceso, no de modernización de la educación, como se hubiera querido, sino en la posibilidad de que los formados y titulados, como los carentes de esta situación, aportaron en la construcción, reconstrucción y deconstrucción de espacios tanto físicos como pedagógicos y afectivos, única alternativa de la educación rural a lo largo del período 1946-1994.

\section{FUENTES}

Decreto 0192 de enero 30 de 1951. Por el cual se adopta el plan de estudios para los Institutos de Enseñanza Pedagógica y se dictan otras disposiciones sobre Educación Normalista.

Decreto 2617 de Septiembre 29 de 1959. Por el cual se reorganiza el sistema de Enseñanza Normalista y se inicia el Plan de Formación de Maestros.

Decreto 1710 de Julio 25 de 1963. Por el cual se adopta el Plan de Estudios de la Educación Primaria Colombiana y se dictan otras disposiciones.

Decreto 1955 de Septiembre 2 de 1963. Por el cual se organiza la educación normalista.

Decreto 080 de Enero 22 de 1974. Por el cual se deroga el Decreto número 045 de 1962 y se dictan otras disposiciones sobre educación media.

Decreto 088 de Enero 22 de 1976. Por el cual se reestructura el sistema educativo y se reorganiza el Ministerio de Educación Nacional.

Decreto 2277 de Septiembre 14 de 1979. Por el cual se adoptan normas sobre el ejercicio de la profesión docente.

Decreto 107 de enero 13 de 1989. Por el cual se reglamentan los Comités DRI.

Decreto 1348 de junio 27 de 1990. Por el cual se crea el Sistema Especial de Formación de Maestros y se dictan otras disposiciones.

Departamento Nacional de Estadística- DANE. Censo de población de 1951.

Ministerio de Educación Nacional. Memorias del Ministro de Educación Nacional (Eliseo Arango), 1949.

Oficina de Educación Iberoamericana. La Educación en el Plano Pedagógica Nacional: Plaza \& Janés editores Colombia S.A, 1959.

\section{REFERENCIA}

Báez Osorio, Myriam. Las Escuelas Normales y el cambio educativo en los Estados Unidos de Colombia en el periodo Radical, 1970-1889. Tunja: Universidad Pedagógica y Tecnológica de Colombia, 2004.

Betancurt Mejía, G. (Compilador). Documentos para la historia del planeamiento de la educación. Informe para el proyecto del primer plan quinquenal (Colombia 1956). Vol. II. Bogotá: Universidad Pedagógica Nacional, 1984.

Díaz V., M. y Chávez B, Ca. Las reformas de las escuelas normales un área de conflicto. Revista Educación y Cultura, No 20 (1990): 15-26. 
Escobar, G. Notas históricas de las Escuelas Normales y las Facultades de Educación en Colombia. Revista Educación y Cultura, No. 20 (1990): 9-23.

Filmus, Daniel. (Compilador) "Las transformaciones educativas en Iberoamérica. Tres desafios: desarrollo e integración". Buenos Aires: OEI- Troquel, 1998.

Flórez Ocho A, y J. E Batista. "Modelos pedagógicos y formación de maestros". Revista Educación y Cultura, No. 7 (1986): 12-18.

Foucault, Michel. "Diálogo sobre el discurso". En: Estética, ética y hermenéutica, editado por Michel FOUCAULT. (Barcelona: Paidós, 1999), 59.

Grupo Pedagógico de Ubaté. "Un ejemplo de autoformación permanente". Revista Educación y Cultura, No. 7 (1986): 38.

Helg, A. La Educación en Colombia: 1918-1957. Bogotá: Universidad Pedagógica Nacional, 1987.

Martínez Boom, A. De la escuela expansiva a la escuela competitiva. Dos modos de modernización en América Latina. Barcelona: Antrophos, 2004.

Martínez Boom, A. "Escuela para el aprendizaje o enseñanza para el pensamiento". Revista Educación y Cultura No.13 (1987).

Martínez Boom, A., C. Noguera, y J. Castro, y O. "Reformas de la Enseñanza en Colombia: 1960-1980. Del énfasis didáctico al énfasis curricular”. Revista Educación y Cultura No. 15 (1988): 12-21.

Mercado, Ruth. Bases académicas para el establecimiento de estudios de postgrado en educación. En: Una educación con calidad y equidad, editado por Martínez. Madrid: OEI, 379380.

Rojas de Ferro, $\mathrm{M}^{\mathrm{a}}$ C. "Análisis de una experiencia". Revista Colombiana de Educación, No 10 (1998).

Salazar, María Cristina. "Elementos Pedagógicos para la Educación Primaria en las áreas rurales". Revista Colombiana de Educación, No. 2 (1978).

Suárez Melo, Lilia. Memorias al Congreso. 1982-1986. Bogotá: Ministerio de Educación Nacional.

Torres, Rosa María. "Alternativas dentro de la educación formal: El programa Escuela Nueva en Colombia". Revista Colombiana de Educación No 32.

Vera G, César y PARRA SANDOVAL, Francisco. "Microcentros y formación docente (un estudio exploratorio en el Departamento del Huila)". Revista Colombiana de Educación, No21 (1990): 4.

Azula Barrera, R. Memorias del Ministro de Educación Nacional. Bogotá: Ministerio de Educación Nacional, Departamento de Enseñanza Normalista, (1990).

Gómez Valderrama, P. Memorias del Ministro de Educación Nacional. Tomo I. Bogotá: Ministerio de Educación Nacional, 1964.

ICOLPE “Personal docente del nivel primario”. Serie Estadísticas Internacional. Educación Primaria. Madrid, 1971.

Kaessmann, Walter. "Diagnóstico sobre la situación actual de la Enseñanza de Matemática en la Escuela Primaria Colombiana". En Análisis de una experiencia, editado por María Cristina Rojas de Ferro. Bogotá: Universidad Pedagógica Nacional, 1966. 
Kurmen Rojas, Alcira y Briones, Guillermo. Evaluación de las Escuelas Normales de Colombia. V. 1. Bogotá: MEN-PNUD-UNESCO, 1978.

Kurmen Rojas, Alcira y Briones, Guillermo. Evaluación de las Escuelas Normales de Colombia. V. 3. Bogotá: MEN-PNUD-UNESCO, 1978.

Medrano, L. Informe del Departamento Normalista al Ministro de Educación Nacional. Bogotá: Ministerio de Educación Nacional, 1949.

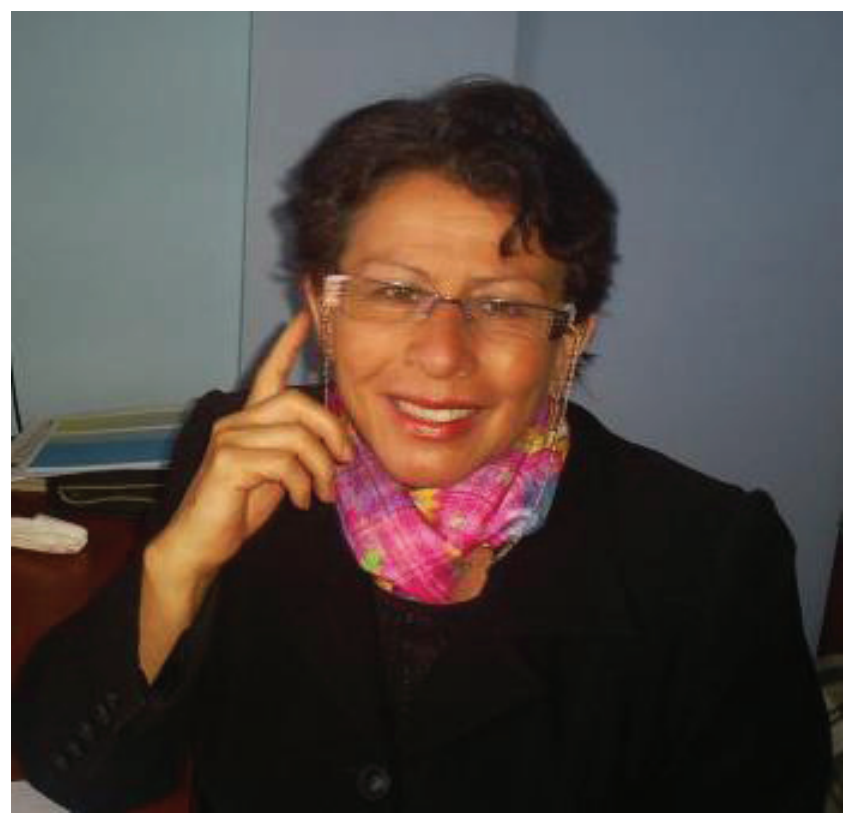

Archivo Personal Alba Nidia Triana Ramírez

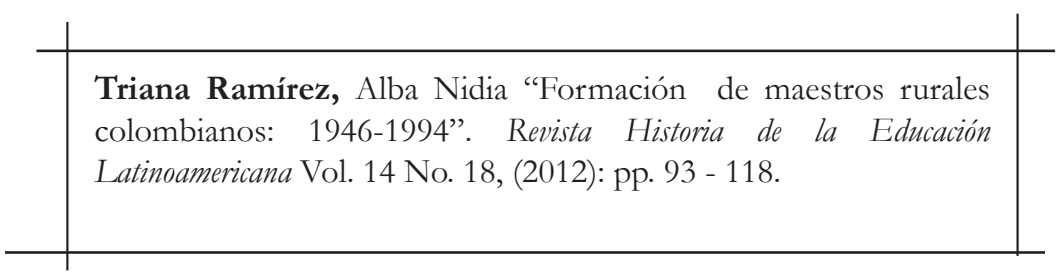

